\title{
A generalized distributed delay model for HBV infection with two modes of transmission and adaptive immunity: A mathematical study
}

\author{
Kalyan Manna ${ }^{1}$ and khalid hattaf ${ }^{2}$ \\ ${ }^{1}$ Indian Institute of Technology Kanpur \\ ${ }^{2} \mathrm{CRMEF}$, Casablanca
}

December 9, 2021

\begin{abstract}
In this paper, we formulate a generalized hepatitis B virus (HBV) infection model with two modes of infection transmission and adaptive immunity, and investigate its dynamical properties. Both the virus-to-cell and cell-to-cell infection transmissions are modeled by general functions which satisfy some biologically motivated assumptions. Furthermore, the model incorporates three distributed time delays for the production of active infected hepatocytes, mature capsids and virions. The well-posedness of the proposed model is established by showing the non-negativity and boundedness of solu- tions. Five equilibria of the model are identified in terms of five threshold parameters R0, R1, R2, R3 and R4. Further, the global stability analysis of each equilibrium under certain conditions is carried out by employing suitable Lyapunov function and LaSalle's invariance principle. Finally, we present an example with numerical simulations to il- lustrate the applicability of our study. Nonetheless, the results obtained in this study are valid for a wide class of HBV infection models.
\end{abstract}

\section{Hosted file}

Generalized model with immunity.pdf available at https://authorea.com/users/423508/articles/ 548735-a-generalized-distributed-delay-model-for-hbv-infection-with-two-modes-oftransmission-and-adaptive-immunity-a-mathematical-study 